\title{
A CONVERSE OF THE GALE-KLEE-ROCKAFELLAR THEOREM: CONTINUITY OF CONVEX FUNCTIONS AT THE BOUNDARY OF THEIR DOMAINS
}

\author{
EMIL ERNST
}

(Communicated by Thomas Schlumprecht)

\begin{abstract}
Given $x_{0}$, a point of a convex subset $C$ of a Euclidean space, the two following statements are proven to be equivalent: (i) every convex function $f: C \rightarrow \mathbb{R}$ is upper semi-continuous at $x_{0}$, and (ii) $C$ is polyhedral at $x_{0}$. In the particular setting of closed convex functions and $F_{\sigma}$ domains, we prove that every closed convex function $f: C \rightarrow \mathbb{R}$ is continuous at $x_{0}$ if and only if $C$ is polyhedral at $x_{0}$. This provides a converse to the celebrated Gale-Klee-Rockafellar theorem.
\end{abstract}

\section{INTRODUCTION}

One basic fact about real-valued convex functions on Euclidean spaces is that they are continuous at each point of their domain's relative interior (see for instance [14, Theorem 10.1]).

On the other hand, it is not difficult to define a convex function which is discontinuous at every point of the relative boundary of its domain. As stated by Carter in his treatise "Foundations of Mathematical Economics" [4, page 334]: This is not a mere curiosity. Economic life often takes place at the boundaries of convex sets, where the possibility of discontinuities must be taken into account.

The celebrated Gale-Klee-Rockafellar (GKR) theorem ([6. Theorem 2]; see also [14, Theorem 10.2]) is a major step toward an accurate understanding of continuity properties for convex functions at points belonging to the relative boundary of their domain. This result is particularly meaningful when applied to the class of closed convex functions, as defined in the seminal work of W. Fenchel ([5]).

GKR Theorem. A convex function is upper semi-continuous at every point at which its domain is polyhedral. Accordingly, a closed convex function is continuous at each such point.

Besides its intrinsic interest, this theorem has proved itself a fertile source of applications. Taking one example out of many, let us remark that since a polyhedron is everywhere polyhedral, the GKR theorem proves the ubiquitous mathematical economics and game theory lemma ([2, Theorem 4.2]), which says that each concave function defined on $P_{+}^{n}$, the cone of the vectors from $\mathbb{R}^{n}$ with positive coordinates, is lower semi-continuous.

Received by the editors December 7, 2011 and, in revised form, January 4, 2012 and January 6, 2012.

2010 Mathematics Subject Classification. Primary 52A20; Secondary 52A41, 52B99.

Key words and phrases. Continuity of convex functions, closed convex functions, polyhedral points, conical points, Gale-Klee-Rockafellar theorem, linearly accessible points. 
The GKR theorem also provides powerful tools in establishing continuity of special convex functions issued from particular optimization problems, such as the M-convex and L-convex functions of Murota and Shioura ([13]).

The example of the closed convex function

$$
f: C \rightarrow \mathbb{R}, \quad f(x, y)=\frac{x^{2}}{y} \text { if }(x, y) \neq(0,0) \text { and } f(0,0)=0
$$

defined on the disk

$$
C=\left\{(x, y) \in \mathbb{R}^{2}: x^{2}+(1-y)^{2} \leq 1\right\},
$$

yet discontinuous at the point $(0,0) \in C$, is well-known ([14, page 83]).

Let us remark that the point at which the previously defined function is discontinuous may (inter alia) be characterized as being the limit of a non-constant sequence made of extreme points of the disk. The lemma on page 870 in [6] proves that this is a very general feature; more precisely, its proof can easily be modified in order to state the following result.

Converse GKR Theorem. Let $C$ be a closed and convex subset of $X$, and $x_{0} \in C$ be the limit of a non-constant sequence of extreme points of $C$ (such a point exists if and only if $C$ is not polyhedral at each and every one of its points). Then there exists at least one closed convex function $f: C \rightarrow \mathbb{R}$ which is not continuous at $x_{0}$.

A standard observation proves that if $C$ is conical at some point $x_{0} \in C$, then none of the non-constant sequences of extreme points of $C$ can converge to $x_{0}$. In this respect, the following result by Howe ([8, Proposition 2]) provides an extension of the reciprocal GKR theorem.

Howe's Theorem. Let $C$ be a closed and convex subset of $X$ and $x_{0} \in C$ be a point at which $C$ is not conical. Then there exists at least one closed convex function $f: C \rightarrow \mathbb{R}$ which is not continuous at $x_{0}$.

An obvious limitation of the previous theorem is that Howe's result is bound to the setting of closed domains, and no conclusion can be drawn for the larger class of convex domains over which closed convex functions may be defined (that is, $F_{\sigma}$ convex sets).

Moreover, this result leaves unanswered the decidedly non-trivial question of the continuity of a closed convex function at points at which the domain is conical without being polyhedral (typically the apex of a circular cone). Indeed, the hypothesis that a closed convex function is automatically continuous at such types of points seems very natural, and this claim has been made (in an implicit form) at least once ([3. Proposition 5, p. 183]). However, this conjecture has been proved false when Goossens ([7, p. 609]) provided a (very elaborate) example of a closed convex function defined on a circular cone and discontinuous at its apex.

This note attempts to fill in the gap between the direct GKR theorem and Howe's result by proving (Theorem 2.4, Section 2) the following statement.

Second Converse GKR Theorem. Given $C$, a convex subset of the Euclidean space $X$, and $x_{0}$, a point at which $C$ is not polyhedral, then there is a convex function $f: C \rightarrow \mathbb{R}$ which is not upper semi-continuous at $x_{0}$.

When, in addition, $C$ is a $F_{\sigma}$ set, then there is $f: C \rightarrow \mathbb{R}$, a closed convex function which is discontinuous at $x_{0}$. 
1.1. Definitions and notation. Let us consider $X$, a Euclidean space endowed with the usual topology, and let us set $x \cdot y$ for the scalar product between the vectors $x$ and $y$ of $X$, and $\|\cdot\|$ for the associated norm.

Given $A$ a subset of $X$, let $X_{A}$ be its affine span (that is, the intersection of all the hyperplanes of $X$ containing $A$ ). The relative boundary of $A$ is defined by the formula

$$
r \partial(A)=\bar{A} \cap \overline{X_{A} \backslash A},
$$

where a superposed bar denotes the closure of a set, while the relation

$$
r i(A)=A \backslash r \partial(A)
$$

defines the relative interior of the set $A$. Let us recall ([14, Theorem 6.2, p. 45]) that the relative interior of a non-empty convex set is non-empty.

As is customary, a subset $A$ of $X$ is said to be an $F_{\sigma}$ set if it is the countable union of a family of closed subsets of $X$ :

$$
A=\bigcup_{i=1}^{\infty} A_{i}, \quad A_{i}=\overline{A_{i}} \forall i \in \mathbb{N} .
$$

Obviously, a closed and convex set is $F_{\sigma}$. In order to provide an example of a convex subset of $X$ which is not $F_{\sigma}$, let us recall ([1, exercise 145, p. 103, and exercise 269, p. 145]) that the set of the irrationals is not $F_{\sigma}$. By homeomorphism, the same holds in $\mathbb{R}^{2}$ for the set of points on the unit circle possessing an irrational angular coordinate.

On the other hand, it is an easy exercise to show that the union between an open disk and an arbitrary subset of its boundary is convex. Therefore, the subset of $\mathbb{R}^{2}$ obtained by putting together the open unit disk and all the points of its boundary possessing an irrational angular coordinate is a convex set but not an $F_{\sigma}$ one.

A function $f: A \rightarrow \mathbb{R}$ is called closed if its epigraph

$$
\text { epi } f=\{(x, r) \in A \times \mathbb{R}: f(x) \leq r\}
$$

is a closed subset of $X \times \mathbb{R}$. Let us notice that the domain of a closed function is necessarily an $F_{\sigma}$ set. The function $f: A \rightarrow \mathbb{R}$ is upper semi-continuous at $x_{0}$ if

$$
f\left(x_{0}\right) \geq \limsup _{x \in A, x \rightarrow x_{0}} f(x) .
$$

In this article, by a polyhedron we mean a set obtained as the intersection of a finite family of closed half-spaces of $X$; accordingly, polyhedra are closed convex sets, not always bounded. Following Klee ([10, p. 86]), we call the set $A$ polyhedral at $x_{0} \in A$ if there are $U$, a neighborhood of $x_{0}$, and $B$, a polyhedron, such that

$$
A \cap U=A \cap B .
$$

Similarly, we call a set $A$ conical at $x_{0} \in A$ if there are $U$, a neighborhood of $x_{0}$, and $K$, a shifted closed convex cone (meaning that 0 is not necessarily its apex), such that

$$
A \cap U=A \cap K .
$$

In other words (Howe, [8, p. 1198]), "near $x_{0}$, the set $A$ looks like a $[\ldots]$ cone." Obviously, a convex set is polyhedral at each point of its relative interior. Moreover, if a set is polyhedral at some point, it is also conical at the same point, but the converse does not generally hold. 


\section{Continuity of COnVEX functions at points of the Relative BOUNDARY OF THEIR DOMAIN}

A key step in proving our main result is provided by Theorem 2.2. This result features a geometrical property of points belonging to the relative boundary of a convex set, which, to the best of our knowledge, has never been addressed.

Following Klee ([9, p. 448]), we call a point $x \in X$ linearly accessible from the subset $A$ of $X$ if there is a point $a$ such that the half-open segment $[a ; x$ [ is contained in $A$. Of course, the linearly accessible points belong to the closure of $A$, but the converse does not generally hold.

For convex sets, however, all points of the closure are linearly accessible (an obvious application of the fact that their relative interior is always non-empty). Theorem 2.2 addresses the question of the linear accessibility of the boundary points for sets which can be expressed as the difference between two convex sets.

Let us first establish to what extent studying this topic helps to demonstrate the converse GKR theorem.

Proposition 2.1. Let $C$ be a subset of $X$ and $x_{0}$ one of its points, and assume that there is a closed convex set $D$ containing $x_{0}$ such that $x_{0} \in \overline{C \backslash D}$, yet $x_{0}$ is not linearly accessible from $C \backslash D$.

i) If $C$ is convex, then there is a convex function $f: C \rightarrow \mathbb{R}$ which is not upper semi-continuous at $x_{0}$.

ii) If $C$ is a $F_{\sigma}$ convex set, then it is possible to find a closed convex function $f: C \rightarrow \mathbb{R}$ which is not continuous at $x_{0}$.

Proof of Proposition 2.1. Without loss of generality, we can assume that $x_{0}=0$. Let us consider the cone spanned by $D$,

$$
\mathcal{C}_{D}=\{x \in X: \lambda x \in D \text { for some } \lambda>0\},
$$

and $\mu_{D}: \mathcal{C}_{D} \rightarrow \mathbb{R}$, the Minkowski gauge of $D$,

$$
\mu_{D}(x)=\inf \left\{\gamma>0: \frac{1}{\gamma} x \in D\right\} .
$$

It is clear that $\mathcal{C}_{D}$ is a convex cone and $\mu_{D}(0)=0$. Moreover, it is well-known (see for instance [14, Corollary 9.7.1, p. 79]) that $\mu_{D}$ is a closed convex function.

We claim that $C \subset \mathcal{C}_{D}$ and that the restriction

$$
f: C \rightarrow \mathbb{R}, \quad f(x)=\mu_{D}(x)
$$

of $\mu_{D}$ to $C$ fulfills point $i$ ) in Proposition 2.1

Indeed, let $x \in C$. As 0 is not linearly accessible from $C \backslash D$, it follows in particular that the segment $[x ; 0[$ is not entirely contained in $C \backslash D$, and since $[x ; 0[\subset C$, it results that

$$
\lambda x \in D \text { for some } 0<\lambda<1 \text {; }
$$

that is, $x \in \mathcal{C}_{D}$. Hence, $C \subset \mathcal{C}_{D}$.

To the end of analyzing the upper semi-continuity of the function $f$ at 0 , let us recall, on one hand, that $f(0)=0$ and, on the other, that the point 0 belongs to the closure of the set $C \backslash D$. One can thus find a sequence, say $\left(x_{n}\right)_{n \in \mathbb{N}}$, of elements from $C \backslash D$ converging to 0 . Pick any of the vectors $x_{n}$. As it does not belong to $D$, the definition of the Minkowski gauge implies that $f\left(x_{n}\right) \geq 1$. The lack of upper semi-continuity of $f$ at 0 is therefore established. 
In order to address point $i$ ) of Proposition 2.1, let us state the standard convex analysis result saying that, given $C$ a convex $F_{\sigma}$ set, there exists at least one closed convex function $g: C \rightarrow \mathbb{R}$. Indeed, $C$ may be expressed as the union of an increasing sequence of convex compact sets, say $\left(A_{n}\right)_{n \in \mathbb{N}}$, and we may set $g$ for the convex envelope of the function

$$
h: C \rightarrow \mathbb{R}, \quad h(x)=\left(\min \left\{n \in \mathbb{N}: x \in A_{n}\right\}\right)^{2} .
$$

Obviously, $g$ is defined on the convex set $C$. Moreover, the technique used by Klee to prove [10. Theorem 4.1] may be used with virtually no modifications to establish that $g$ is closed.

If the function $g$ is discontinuous at 0 , then it fulfills point $i i$ ). Assume now that the function $g$ is continuous at 0 . The application

$$
f: C \rightarrow \mathbb{R}, \quad f(x)=g(x)+\mu_{D}(x)
$$

is closed and convex as the sum of closed and convex functions. Moreover, $f$ is the sum between a function which is continuous at 0 and a function which is discontinuous at the same point. Thus $f$ is a closed convex application discontinuous at 0 , and Proposition 2.1 is completely proved.

With the conclusions of Proposition 2.1 in mind, let us address Theorem 2.2, the most technical part of our paper.

Theorem 2.2. Let $C$ be a convex subset of $X$, and $x_{0}$ be one of its points. The two following statements are equivalent:

i) $C$ is not polyhedral at $x_{0}$.

ii) There is a closed convex set $D$ containing $x_{0}$ such that $x_{0} \in \overline{C \backslash D}$, yet $x_{0}$ is not linearly accessible from $C \backslash D$.

Proof of Theorem 2.2. Without loss of generality, we can assume that $x_{0}=0$.

$i) \Rightarrow$ ii) Let us assume that $C$ is not polyhedral at 0 . By virtue of Corollary 3.3 in $([10$, p. 88$])$, it results that the same holds for $\mathcal{C}_{C}$, the convex cone spanned by $C$. Let us first prove a general result on non-polyhedral cones.

Lemma 2.3. Let $E$ be a non-polyhedral convex cone with apex 0 . Then there is a sequence $\left(y_{n}\right)_{n \in \mathbb{N}} \subset X$ such that:

i) for each $x \in E$ and $n$ large enough, the sequence $\left(x \cdot y_{n}\right)_{n \in \mathbb{N}}$ takes only nonpositive values;

ii) for each $n \in \mathbb{N}$, there is $x_{n} \in E$ such that $x_{n} \cdot y_{n}>0$.

Proof of Lemma 2.3. A far-reaching characterization of polyhedrality for cones was achieved by Klee ([10, Theorem 4.11, p. 92]; the particular case of closed convex cones had previously been provided by Mirkil [12, Theorem, p. 1]), which says that a convex cone is polyhedral if and only if its projection on every two-dimensional affine manifold of $X$ is a closed set.

Accordingly, the convex cone $\Pi(E)$ is not closed, where $\Pi: X \rightarrow X_{1}$ is the operator of projection onto some plane $X_{1}$ of $X$. Let $v$ be a vector belonging to the closure of $\Pi(E)$ but not to $\Pi(E)$ itself (as $0=\Pi(0)$, it follows that $v \neq 0$ ).

As the relative interiors of a convex set and of its closure coincide, the fact that the vector $v$ belongs to $\overline{\Pi(E)} \backslash \Pi(E)$ implies that $v$ lies within the relative boundary of $\overline{\Pi(E)}$. A standard supporting hyperplane argument shows that there exists an 
element $w \in X_{1}$ such that the function $x \rightarrow x \cdot w$ achieves its maximum over $\overline{\Pi(E)}$ at $v$. On one hand, this fact implies that

$$
\Pi(x) \cdot w \leq v \cdot w \quad \forall x \in E ;
$$

on the other, since both the vectors 0 and $2 v$ belong to $\overline{\Pi(E)}$, it yields that

$$
0=0 \cdot w \leq v \cdot w, \quad 2 v \cdot w \leq v \cdot w .
$$

Hence

$$
v \cdot w=0 \text {. }
$$

For every $n \in \mathbb{N}$, let us set $y_{n}=w+\frac{v}{n}$. As $y_{n} \in X_{1}$, it results that

$$
x \cdot y_{n}=\Pi(x) \cdot\left(w+\frac{v}{n}\right) \quad \forall x \in E, n \in \mathbb{N} .
$$

We claim that the sequence $\left(y_{n}\right)_{n \in \mathbb{N}}$ fulfills relation $i$ ). Let us pick $x \in E$. In view of relation (2.1), there are two possible cases: a) $\Pi(x) \cdot w<v \cdot w$ and $b$ ) $\Pi(x) \cdot w=v \cdot w$.

In case $a$ ), from relation (2.2) we infer that

$$
\Pi(x) \cdot w<0 .
$$

As obviously

$$
\lim _{n \rightarrow \infty} \Pi(x) \cdot \frac{v}{n}=0,
$$

statement $i$ ) comes from relations (2.3), (2.4) and (2.5).

In case $b), \Pi(x) \cdot w=0$, so $\Pi(x)$ belongs to the hyperplane

$$
H_{w}=\left\{z \in X_{1}: z \cdot w=0\right\}
$$

of $X_{1}$. But $X_{1}$ is a two-dimensional vector space, and each of its hyperplanes is in fact a line; moreover, we have already proved (relation (2.2) ) that the non-null vector $v$ belongs to $H_{w}$. It results that $\Pi(x)$ lies on the line $\mathbb{R} v$.

As $v \notin \Pi(E)$ and since $\Pi(E)$ is a cone with apex 0 , it follows that the half-line $\mathbb{R}_{+}^{*} v$ is disjoint from $\Pi(E)$. We may thus affirm that

$$
\Pi(x)=-\lambda v \text { for some } \lambda \geq 0 .
$$

By combining relations (2.2), (2.3) and (2.6), we conclude that

$$
x \cdot y_{n}=-\lambda \frac{\|v\|^{2}}{n} \leq 0 \quad \forall n \in \mathbb{N} .
$$

Statement $i$ ) is therefore fulfilled in both situations $a$ ) and $b$ ).

Let us now address relation $i i)$. As $v \in \overline{\Pi(E)}$, there is a sequence $\left(z_{n}\right)_{n \in \mathbb{N}} \subset E$ such that the sequence $\left(\Pi\left(z_{n}\right)\right)_{n \in \mathbb{N}}$ converges to $v$. Pick $k \in \mathbb{N}$ and apply relation (2.3) for $x=z_{n}$ and $y_{k}$ :

$$
z_{n} \cdot y_{k}=\Pi\left(z_{n}\right) \cdot\left(w+\frac{v}{k}\right) \quad \forall n \in \mathbb{N} .
$$

Accordingly,

$$
\lim _{n \rightarrow \infty}\left(z_{n} \cdot y_{k}\right)=v \cdot\left(w+\frac{v}{k}\right) ;
$$

by virtue of relation (2.2), we obtain that

$$
\lim _{n \rightarrow \infty}\left(z_{n} \cdot y_{k}\right)=\frac{\|v\|^{2}}{k}>0 .
$$


The set $L_{k}=\left\{n \in \mathbb{N}: z_{n} \cdot y_{k}>0\right\}$ is therefore non-empty. Set $u(k)=\min L_{k}$, and, for each positive integer $n$, define $x_{n}$ as being $z_{u(n)}$. The sequences $\left(x_{n}\right)_{n \in \mathbb{N}}$ and $\left(y_{n}\right)_{n \in \mathbb{N}}$ obviously fulfill relation $\left.i i\right)$.

Let us now get back to the proof of the implication $i) \Rightarrow i i$ ) from Theorem 2.2 and apply the conclusions of Lemma 2.3 to the non-polyhedral cone $\mathcal{C}_{C}$.

Accordingly, there are two sequences, $\left(x_{n}\right)_{n \in \mathbb{N}} \subset \mathcal{C}_{C}$ and $\left(y_{n}\right)_{n \in \mathbb{N}} \subset X$, such that

$$
\forall x \in \mathcal{C}_{C}, x \cdot y_{n} \leq 0 \text { for } n \text { large enough, }
$$

and

$$
x_{n} \cdot y_{n}>0 \quad \forall n \in \mathbb{N} .
$$

Since $x_{n}$ is a vector from the cone spanned by the convex set $C$ and $0 \in C$, we find that there is a positive real number, say $\zeta_{n}$, such that

$$
\lambda x_{n} \in C \quad \forall 0 \leq \lambda \leq \zeta_{n} .
$$

Set

$$
\lambda_{n}=\min \left(\zeta_{n}, \frac{1}{n\left\|x_{n}\right\|}\right) \quad \forall n \in \mathbb{N} .
$$

It follows that $\lambda_{n} x_{n} \in C$ for each positive integer $n$ and that the sequence $\left(\lambda_{n} x_{n}\right)_{n \in \mathbb{N}}$ converges to 0 .

Define the set

$$
D=\left\{x \in X: x \cdot y_{n} \leq \lambda_{n} \frac{x_{n} \cdot y_{n}}{2} \forall n \in \mathbb{N}\right\} .
$$

Obviously, $D$ is a closed convex set which contains the point 0 . Moreover, for every positive integer $n$, the point $\lambda_{n} x_{n}$ belongs to $C$ but does not belongs to $D$. As the sequence $\lambda_{n} x_{n}$ converges to 0 , we may conclude that $0 \in \overline{C \backslash D}$.

To show that 0 is not linearly accessible from $C \backslash D$, let us pick $x \in C$ and recall (see relation (2.7) ) that the sequence $\left(x \cdot y_{n}\right)_{n \in \mathbb{N}}$ takes only a finite number of positive values, while, by virtue of the inequality (2.8), the sequence $\left(\lambda_{n} \frac{x_{n} \cdot y_{n}}{2}\right)_{n \in \mathbb{N}}$ has only positive terms. Thus, the sequence $\left(\frac{2\left(x \cdot y_{n}\right)}{\lambda_{n}\left(x_{n} \cdot y_{n}\right)}\right)_{n \in \mathbb{N}}$ has a finite number of positive values, so there is a positive real number $a$ such that

$$
\frac{2\left(x \cdot y_{n}\right)}{\lambda_{n}\left(x_{n} \cdot y_{n}\right)} \leq a \quad \forall n \in \mathbb{N} .
$$

For every positive number $\mu$ such that $\mu a \leq 1$, it results that

$$
\mu\left(x \cdot y_{n}\right) \leq(\mu a) \lambda_{n} \frac{x_{n} \cdot y_{n}}{2} \leq \lambda_{n} \frac{x_{n} \cdot y_{n}}{2} \quad \forall n \in \mathbb{N} .
$$

Accordingly,

$$
\mu x \in D \quad \forall 0 \leq \mu \leq \frac{1}{a},
$$

and we may conclude that there is no point $x \in C$ such that the segment $[x, 0[$ is entirely contained within $C \backslash D$. In other words, the point 0 is not linearly accessible from $C \backslash D$.

ii) $\Rightarrow i$ ) This implication easily follows by combining the classical GKR theorem and Proposition 2.1 .

The main result of this note now stems from combining Proposition 2.1 and Theorem 2.2 
Theorem 2.4. Given $C$, a convex subset of the Euclidean space $X$, and $x_{0} \in C$, every convex function $f: C \rightarrow \mathbb{R}$ is upper semi-continuous at $x_{0}$ if and only if $C$ is polyhedral at $x_{0}$.

When, in addition, $C$ is an $F_{\sigma}$ set, then each closed convex function $f: C \rightarrow \mathbb{R}$ is continuous at $x_{0}$ if and only if $C$ is polyhedral at $x_{0}$.

\section{ACKNOWLEDGMENT}

The author is grateful to the anonymous referee for helpful comments and suggestions, which have been included in the final version of this paper.

\section{REFERENCES}

1. A. V. Arkhangel'skiı̌, V. I. Ponomarev, Fundamentals of general topology: problems and exercises, Mathematics and Its Applications, 13. Dordrecht - Boston - Lancaster, 1984. MR785749 (87i:54001)

2. M. Avriel, W. E. Diewert, S. Schaible, I. Zang, Generalized concavity, Classics in Applied Mathematics, 63, Philadelphia, 2010. MR0927084 (89e:90155)

3. J. Bair, Liens entre le cône d'ouverture interne et l'internat du cône asymptotique d'un convexe, Bull. Soc. Math. Belg., Sŕ. B 35 (1983), 177-187. MR731806 (85f:52009)

4. M. Carter, Foundations of mathematical economics, MIT Press, Cambridge, 2001. MR.1865841 (2002i:91001)

5. W. Fenchel, On conjugate convex functions, Can. J. Math. 1 (1949), 73-77. MR0028365 (10:435b)

6. D. Gale, V. Klee, R. T. Rockafellar, Convex functions on convex polytopes, Proc. Am. Math. Soc. 19 (1968), 867-873. MR0230219(37:5782)

7. P. Goossens, Hyperbolic sets and asymptotes, J. Math. Anal. Appl. 116 (1986), 604-618. MR842825 (87m:46024)

8. R. Howe, Automatic continuity of concave functions, Proc. Am. Math. Soc. 103 (1988), 11961200. MR955008 (89g:90164)

9. V. Klee, Convex sets in linear spaces, Duke Math. J. 18 (1951), 443-466. MR0044014 $(13: 354 \mathrm{f})$

10. V. Klee, Some characterizations of convex polyhedra, Acta Math. 102 (1959), 79-107. MR0105651 (21:4390)

11. P. Maćkowiak, Some remarks on lower hemicontinuity of convex multivalued mappings, Econ. Theory 28 (2006), 227-233. MR2217897(2006k:54023)

12. H. Mirkil, New characterizations of polyhedral cones, Canad. J. Math. 9 (1957), 1-4. MR0083761 (18:757d)

13. K. Murota, A. Shioura, Note on the continuity of $M$-convex and L-convex functions in continuous variables, J. Oper. Res. Soc. Japan 51 (2008), 265-273. MR2492676 (2010f:26015)

14. R. T. Rockafellar, Convex analysis, Princeton University Press, $1968 . \quad$ MR.1451876 $(97 \mathrm{~m}: 49001)$

UMR 6632, Aix-Marseille University, Marseille, F-13397, France

E-mail address: Emil.Ernst@univ-amu.fr 